\title{
Group Theoretical Route to Deterministic Weyl Points in Chiral Photonic Lattices
}

\author{
Matthias Saba, ${ }^{1, *}$ Joachim M. Hamm, ${ }^{1}$ Jeremy J. Baumberg, ${ }^{2}$ and Ortwin Hess ${ }^{1, \dagger}$ \\ ${ }^{1}$ The Blackett Laboratory, Imperial College London, London SW7 2AZ, United Kingdom \\ ${ }^{2}$ The Cavendish Laboratory, University of Cambridge, Cambridge CB3 OHE, United Kingdom
}

(Received 5 June 2017; published 30 November 2017)

\begin{abstract}
Topological phases derived from point degeneracies in photonic band structures show intriguing and unique behavior. Previously identified band degeneracies are based on accidental degeneracies and subject to engineering on a case-by-case basis. Here we show that deterministic pseudo Weyl points with nontrivial topology and hyperconic dispersion exist at the Brillouin zone center of chiral cubic symmetries. This conceivably allows realization of topologically protected frequency isolated surface bands in 3D and $n=0$ properties as demonstrated for a nanoplasmonic system and a photonic crystal.
\end{abstract}

DOI: 10.1103/PhysRevLett.119.227401

Current broad interest in topological phases, triggered by the discovery of the quantum Hall effect [1] and its theoretical investigation [2-4], can mainly be attributed to the fact that topological features are, due to their discrete nature, insensitive to system perturbations, and can, for example, give rise to the existence of topologically induced unidirectional edge states for bulk systems $[5,6]$. Plasmonic [7] and single electron [8] surface states of Weyl semimetals, with an isolated point degeneracy of nontrivial topology, are known to be stable against perturbations and to give rise to peculiar electron dynamics. Recently, it has been demonstrated that topological quantization occurs in entirely classical systems such as two-dimensional (2D) photonic crystals $[9,10]$, sparking a new wave of research on photonic topology [11]. In particular, topologically protected Weyl points with hyperconic dispersion have been found in double gyroid photonic crystals with broken parity-time symmetry [12], and more recently in a 2D photonic time crystal [13]. Concurrently, group theory provides a tool to predict whether a given spatiotemporal symmetry permits or deterministically induces topologically nontrivial point degeneracies, also know as exceptional points, associated with an algebraic singularity in the band structure. This idea has successfully found its way and been applied to classical $[14,15]$ and quantum mechanical $[16,17]$ systems. Indeed, group theory predicts the existence of deterministic two- and threefold degeneracies for cubic symmetries at the center of the Brillouin zone (BZ), also know as the $\Gamma$ point [18].

Here we show on the basis of group and perturbation theory that symmetry induced threefold degenerate pseudoWeyl points (PWPs) at the $\Gamma$ point split isotropically in first

Published by the American Physical Society under the terms of the Creative Commons Attribution 4.0 International license. Further distribution of this work must maintain attribution to the author(s) and the published article's title, journal citation, and DOI. order in $\boldsymbol{k}$ for any chiral cubic space group with time reversal symmetry. We predict and demonstrate that the identified PWPs exhibit a nontrivial topology, leading to protected surface states. They exist in entirely classical systems, constituting a deterministic 3D analog to previously studied accidental Dirac points [19], and are comparable to similar degeneracies in electronic band structures $[17,20]$. The predictive potential of the theory is corroborated on the basis of a specific model structure.

In this Letter, we first derive a $3 \mathrm{D}$ perturbation Hamiltonian that leads to hyperconic dispersion with nontrivial topology, and an intermediate flat band. We then construct a minimalistic geometry, a $P 2_{1} 3$ sphere packing (Fig. 1), which satisfies the symmetry requirements, and apply it to a quasistatic coupled-dipole model, before discussing topologically protected surface states that emanate from a PWP in a photonic crystal analog. This underscores that the existence of PWPs, including the peculiar transport properties of associated bulk and surface states, only depends on the underlying symmetry irrespective of the particular physical realization.

Our theory applies to all linear and self-consistent physical systems with time reversal invariance and chiral cubic symmetry. First order degenerate perturbation theory and representation theory, the latter of which provides the selection rules for the matrix elements within the former, predict the band structure at deterministic points of degeneracy; for details of the derivation we refer to the Supplemental Material [22], which includes Refs. [18,21,23-33]. For all deterministic threefold degeneracies at $\Gamma$, this procedure yields a perturbation matrix

$$
W_{\alpha \beta}(\boldsymbol{k})=\imath d \sum_{\gamma} \epsilon_{\alpha \beta \gamma} k_{\gamma},
$$

valid for small $k \ll 2 \pi / a$ (with lattice constant $a$ ), with a free parameter $d \in \mathbb{R}$ and $\alpha, \beta, \gamma$ iterating over the three partners of the $k=0$ irreducible representation of the space group that span the degenerate eigenspace. Note that 
(a)

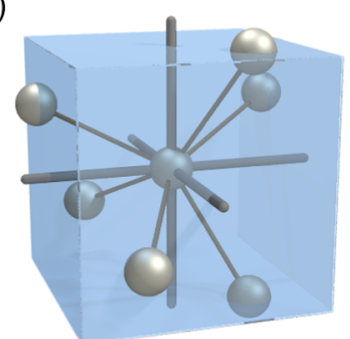

(c)

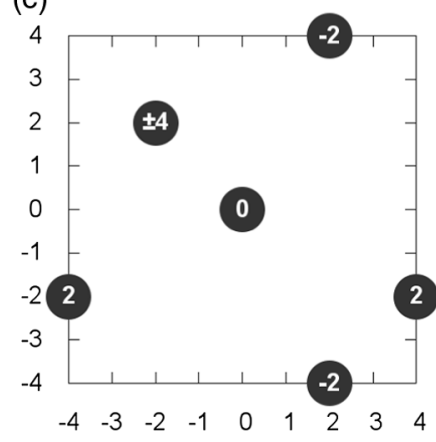

(d)

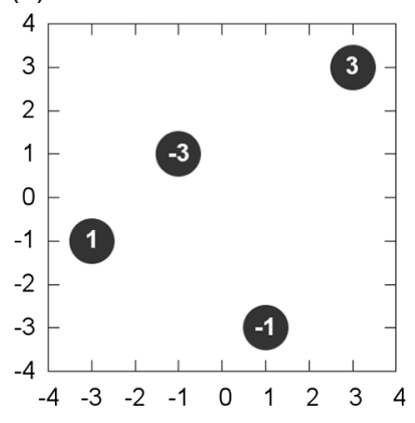

FIG. 1. Illustration of the $P 2{ }_{1} 3$ sphere packing. (a) The glass cube shows the simple cubic unit cell, that is centred at the position of one of the spheres, whose 6 nearest neighbors lie on the cubes' facets. The thick Cartesian rods, and the thin connection rods are shown for illustration purposes only. (b) The same cube shown from the [111] direction. (c) Projection of (a) onto the [001] plane, with spatial unit $a / 8$ and $z$ coordinate in the respective sphere. (d) Same as (c), but with crystallographic choice of origin [21].

Eq. (1) closely resembles an isotropic Weyl Hamiltonian $\mathcal{W}_{i j}=d \sum_{\gamma} \sigma_{i j, \gamma} k_{\gamma}$, with the Pauli matrices $\sigma_{i j, \gamma}(i, j \in\{1,2\})$ here substituted by the 3D Levi-Civita tensor $\epsilon_{\alpha \beta \gamma}$. The first order perturbation eigenfrequencies corresponding to $W(\boldsymbol{k})$ are $k_{0,1}:=\omega_{1} / c=\{0, \pm d k\}$ (with speed of light $c$ ); they only depend on the absolute value of $\boldsymbol{k}$ and describe isotropic hyperconic dispersion.

We thus define a PWP as the degeneracy point $\left(0, k_{0,0}\right)$ at which the pair of hypercones $\left(\boldsymbol{k}_{1}, k_{0,0} \pm d k_{1}\right)$ in the four dimensional $\left(\boldsymbol{k}, k_{0}\right)$ parameter space meets. The flat band modes correspond to longitudinal fields (parallel to $\boldsymbol{k}$ ) for the systems under investigation, qualitatively discriminating its states from those in the hyperconic bands. From a topological perspective, the associated Chern numbers can be analytically calculated for each of the three bands (see the Supplemental Material [22]). They evaluate to $C=0$ for the flat band and $C= \pm 2$ for the two hyperconic bands, showing a nontrivial topological signature, similar to a genuine Weyl point with Chern numbers $C= \pm 1$. Table I compares the key characteristics of genuine Weyl and double Weyl cones, and PWPs.

No twofold degenerate deterministic analogs with hyperconic dispersion exist at the $\Gamma$ point, shown by direct analysis of the 3D space groups [21], with the exception of the trigonal groups $P 312$ (149) and P321 (150): these have

TABLE I. Comparison between genuine Weyl points, double Weyl points, and the pseudo-Weyl points introduced in this manuscript. Only pseudo-Weyl points can be found at the center of the Brillouin zone deterministically and, therefore, lead to exotic bulk phases (e.g., supporting $n=0$ behavior).

\begin{tabular}{lccc}
\hline \hline & Weyl & Double Weyl & Pseudo Weyl \\
\hline Dimension & 2 & 4 & 3 \\
Chern number & \pm 1 & \pm 2 & $\{0, \pm 2\}$ \\
Determinism & $R$, broken $\tau$ & $R$ & $R$ and $\Gamma$ \\
$n=0 ?$ & No & No & Yes \\
\hline \hline
\end{tabular}

two-dimensional representations that split into an anisotropic hypercone if time reversal symmetry is present. Similar points have been observed in a $P 622$ (177) geometry in Ref. [34], albeit not at the $\Gamma$ point. A closely related matter is the nonexistence of deterministic Dirac points at the $\Gamma$ point of two-dimensional crystals, including hexagonal lattices [14]. Furthermore, deterministic PWPs at the center of the Brillouin zone (which provide a route to metamaterials with vanishing refractive index $n=0$ ) require chiral cubic symmetry.

To elucidate the physics (ahead of a concrete experimental realization), we consider an effective plasmonic model consisting of metallic nanospheres of radius $\rho$ in vacuum (as in Refs. [32,35]). The position $\boldsymbol{r}_{i}$ of sphere $i$ shall be such that the distance $d_{i j}=\left|\boldsymbol{r}_{i}-\boldsymbol{r}_{j}\right| \gg \rho$ for any pair of spheres $(i, j)$. In the quasistatic approximation, Maxwell's equations thus take the self-consistent form (with dipole moments $\boldsymbol{p}_{i}$ of the individual spheres) [36]:

$$
\boldsymbol{p}_{i}=\alpha\left(k_{0}\right) \sum_{j \neq i} \mathcal{G}\left(\boldsymbol{r}_{i}-\boldsymbol{r}_{j}, k_{0}\right) \boldsymbol{p}_{j} .
$$

Here, $\alpha\left(k_{0}\right)=\rho^{3}\left(1-3 k_{0}^{2} / k_{p}^{2}\right)^{-1}$ is the polarizability of a metallic sphere in vacuum that is modeled by a nondissipative Drude response with plasma wave number $k_{p}$; $\mathcal{G}\left(\boldsymbol{r}, k_{0}\right)$ is the dyadic Green function for the monochromatic Maxwell wave operator at frequency $k_{0}$.

We arrange the spheres to form a chiral cubic sphere packing with nonsymmorphic $P 22_{1} 3$ (198) symmetry (Fig. 1), which is minimalistic in the sense that it generates a vector space of lowest possible dimension within our model. The index $i$ is thus conveniently substituted by a multi-index $(\boldsymbol{n}, \mu)$, with $\boldsymbol{r}_{\boldsymbol{n}, \mu}=\boldsymbol{T}_{\boldsymbol{n}}+\boldsymbol{r}_{\mu}$ given by the sum of the lattice vector $\boldsymbol{T}_{\boldsymbol{n}}=a \boldsymbol{n}$ and the position within the unit cell $\boldsymbol{r}_{\mu}$. Bloch's theorem then implies for the polarization vectors $\boldsymbol{p}_{\boldsymbol{n}, \mu}=\boldsymbol{p}_{\mu} \exp \left\{\boldsymbol{l} \boldsymbol{k} \cdot \boldsymbol{T}_{\boldsymbol{n}}\right\}$, with the Bloch wave vector $\boldsymbol{k}$ that is a free parameter within the first $\mathrm{BZ}[-\pi / a, \pi / a)^{3}$.

For the particular geometry (Fig. 1), Eq. (2) therefore reduces to a family of low-dimensional nonlinear Hermitian eigenproblems, with eigenvalues $k_{0}$ and dimension $N=12$ :

$$
\alpha^{-1}\left(k_{0}\right) \boldsymbol{p}_{\mu}=\sum_{\nu} M_{\mu \nu}\left(\boldsymbol{k}, k_{0}\right) \boldsymbol{p}_{\nu}
$$




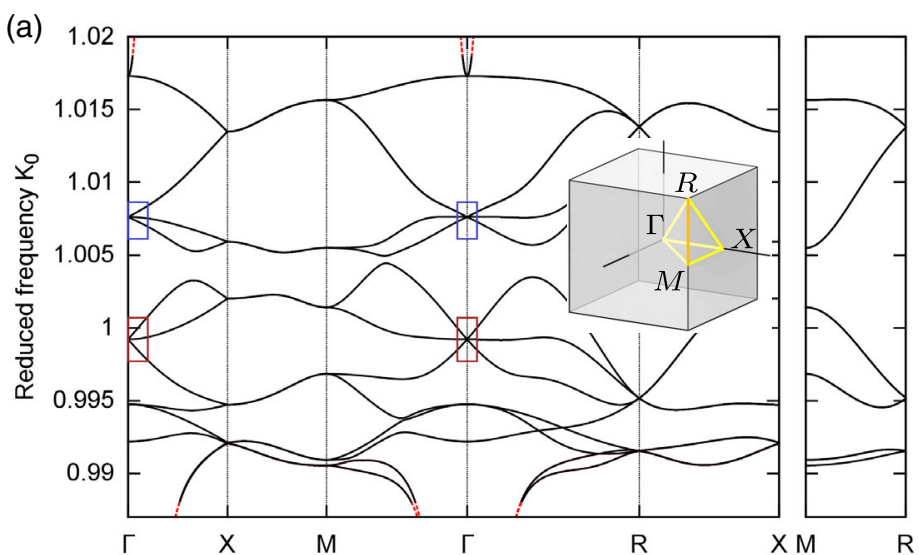

(b)
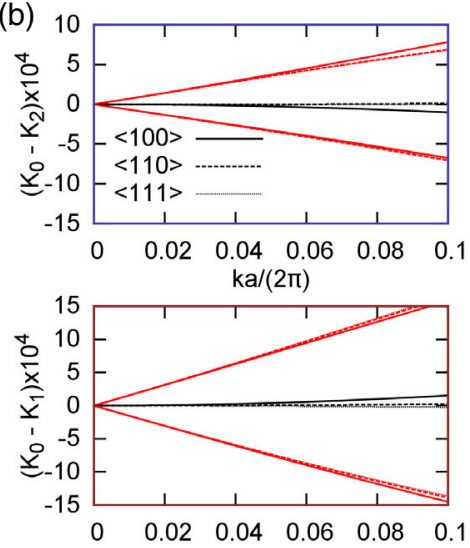

FIG. 2. Band structure of the plasmonic sphere packing illustrated in Fig. 1 for $x / a=0.175, k_{p} a /(2 \pi)=0.1$, and $\rho / a=0.1$. (a) All $N=12$ branches, corresponding to the solutions of Eq. (2). (b) The isolated triplet states that meet at $K_{1}=0.9992$ and $K_{2}=1.0076$ show a particularly clear and isotropic Weyl hypercone (red) and a flat dark mode (black) in between, even for relatively large $k \approx \pi /(5 a)$. The blue (red) boxes highlighted in (a) correspond to the subfigures of the same color in (b).

Since the matrix $M$ generally imposes a small perturbation to the single sphere resonance solution $K_{0}^{(n)}:=\sqrt{3} k_{0}^{(n)} / k_{p}=1$ [due to $\rho^{-3} \gg 1$ in Eq. (3)], the eigenvalue problem is linearized by approximating $M_{\mu \nu}\left(\boldsymbol{k}, k_{0}\right) \approx M_{\mu \nu}\left(\boldsymbol{k}, k_{p} / \sqrt{3}\right)=$ : $M_{\mu \nu}(\boldsymbol{k})$. The above assumption is inaccurate close to the Ewald sphere $k_{0}=|\boldsymbol{k}|$ in the BZ, caused by poles in the diagonal entries of $M\left(\boldsymbol{k}=k_{0} \hat{\boldsymbol{k}}\right)$. This inaccuracy, however, only affects the two modes at the top and the bottom of the band structure on either side of the pole, highlighted by a dashed red line in Fig. 2(a). The eigenvalues $\lambda_{n}(\boldsymbol{k})=\alpha^{-1}\left(k_{0}\right)$ of $M(\boldsymbol{k})$ generate the dispersion relation $K_{0}^{(n)}(\boldsymbol{k})=$ $\left[1-\rho^{3} \lambda_{n}(\boldsymbol{k})\right]^{1 / 2}$, as shown in the band structure diagram in Fig. 2. Figure 2(b), in particular, illustrates an example where the first order perturbation outweighs higher orders even for relatively large Bloch wave number $k \approx \pi /(5 a)$, so that an almost perfect hypercone can be observed. On the other hand, as theoretically predicted, both bands emanating from the twofold degeneracy at $K_{0}=0.995$ in Fig. 2(a) are flat.

The universality of our group theoretical predictions is vividly demonstrated by replacing the metallic spheres by larger spheres of radius $\rho / a=0.25$ (fill fraction $\pi / 12 \approx 26 \%$ ), made of a high refractive index material with $n=4$. We thus construct a photonic crystal of the same symmetry. The associated band structure (calculated with MPB [37]) close to $k_{0} a /(2 \pi)=0.5$ (see the Supplemental Material [22], Fig. I ) resembles Fig. 2. A partial gap opens in the projected bulk band structure with respect to a [001] inclination in Fig. 3(a): this is the blue area of all $\left(\boldsymbol{k}_{\|}, k_{0}\right)$ for which at least one bulk mode exists for arbitrary $k_{z} \in \mathbb{R}$ [33]. Since the PWP and the determinstic double Weyl cone at $R$ (projected onto $A$ ) are protected by cubic symmetry, this gap can apparently be opened by, e.g., perturbing the sphere positions (see the Supplemental Material [22], Fig. II) while maintaining the nontrivial topology and thus producing topological surface states in the complete band gap. In contrast, a topologically protected genuine Weyl point cannot be perturbed to open a complete band gap [12].

Topological surface states exist in the gap at the interface between two enantiomorphic sphere packings (with identical bulk band structure, but opposite Chern characteristics): Figure 3(a) shows the surface mode dispersion of 12 unit cells of a right handed crystal $(x / a=0.175)$ and 12 unit cells of a left handed crystal $(x / a=-0.175)$ stacked in the [001] direction in a supercell configuration. The space group of this geometry is monoclinic with $P 2_{1} / c$ (14) symmetry (note, however, that the Bravais lattice is tetragonal). The inset shows the surface BZ, within the $\boldsymbol{b}_{3}=0$ plane perpendicular to the stacking direction of the supercell, cf. Fig. 16 in Ref. [38]. The supercell symmetry requires all modes along $Z-A-X$ [Fig. 3(a), left inset] to be twofold degenerate. On the other hand, symmetry does not impose degeneracies along $\Gamma-Z$ and $X-\Gamma$ (including $\Gamma$ itself). The surface states are still paired, which can be understood as follows: consider a surface mode along $\Gamma-Z$ that has a field profile that is bound to a single interface. Along this path, $\boldsymbol{k}$ is invariant under the twofold rotation corresponding to the twofold screw axis 2 in $P 2_{1} / c$ [21] (note that our $x$ axis corresponds to their $y$ axis). This screw axis transports the field profile from one interface to the other, so that a $1 \mathrm{D}$ representation requires field intensities of equal magnitude on both interfaces. However, the two interfaces are separated by a zero field bulk region by definition, so that Maxwell's equations are also satisfied for the same frequency by a field profile that is nonzero at one of the two interfaces only. The mode must thus be twofold degenerate. Close to the $\Gamma$ point, the decay length becomes larger than 6 unit cells, so that the second argument becomes invalid for the particular supercell with 12 unit cells per enantiomer. For $X-\Gamma$, the same line of 

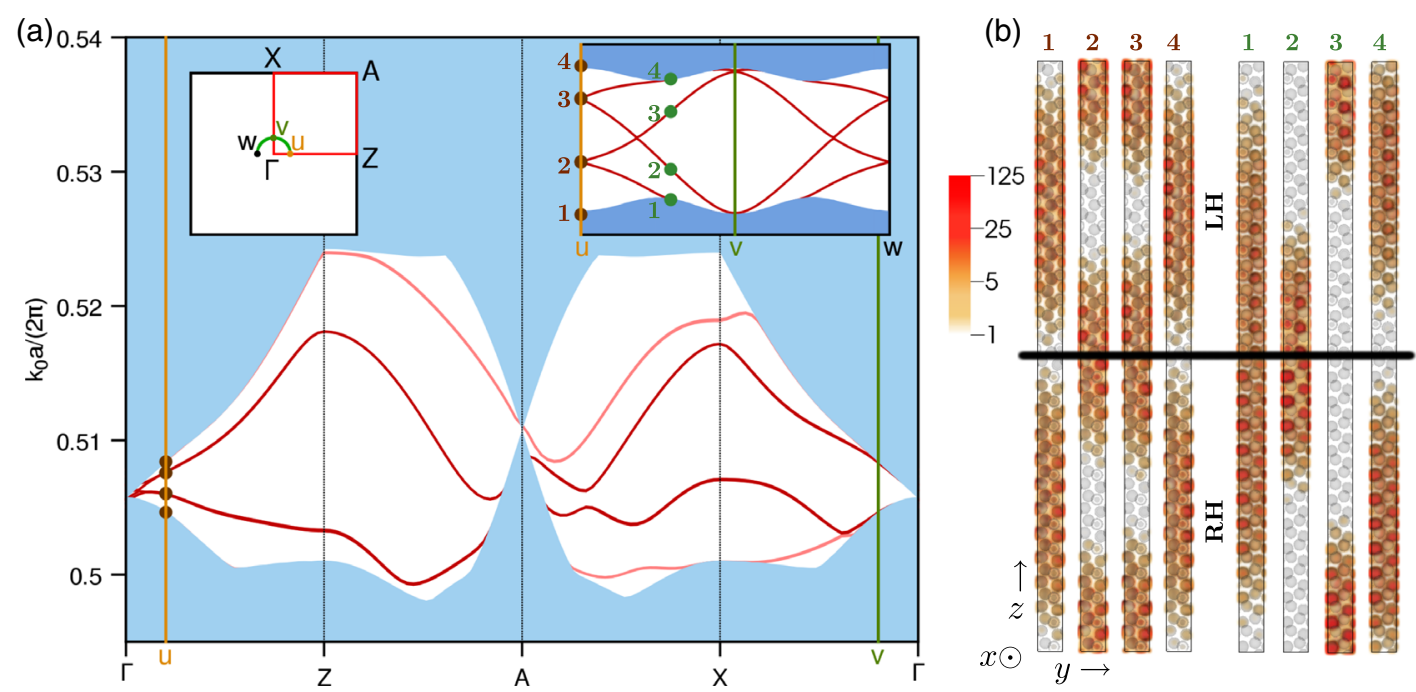

FIG. 3. Surface modes close to a PWP frequency. (a) Surface band structure for a supercell made of, respectively, 12 unit cells of two enantiomorphic sphere packings. Topologically protected (dark red) and unprotected (pale red) surface bands are present within the partial gap of the projected bulk band structure (blue). The main graph shows the band structure along the irreducible BZ boundary (red path, left inset), whereas the inset on the right follows a small semicircle at the $\Gamma$ point (green path, left inset). The individual paths intersect at two points $u$ and $v$. (b) Field energy distribution (arbitrary units) corresponding to points of the same color in (a). Dielectric spheres are shaded for illustration. The interfaces between the right handed (RH) and the left handed (LH) crystal are at the center (black line) and at the end of the unit cell.

thought applies to the glide plane 4 in $P 2_{1} / c$. Figure 3(b) demonstrates that the modes within the bulk gap are indeed localized at the surface, in contrast to other supercell modes within the blue bulk region. The brown points 2 and 3 in Fig. 3(a) highlight two degenerate mode pairs at $\boldsymbol{k}=0.2 \pi / a \times(1,0,0)^{T}$; these split for $\boldsymbol{k}=0.2 \pi / a \times(\cos (\phi), \sin (\phi), 0)^{T}$ with $\phi \neq 0$ (green points for $\phi=0.28 \pi)$.

We have thus shown that surface states exist. But are these also of topological nature? The conventional path $\Gamma-Z-A-X-\Gamma$ does not reveal the topological nature of the surface states emanating from the PWP. To show that these are, indeed, protected, we follow Ref. [39] and consider the cylinder $\boldsymbol{k}\left(\varphi, k_{z}\right)=\left(k \cos (\varphi), k \sin (\varphi), k_{z}\right)^{T}$ (with constant $k$ and $-\pi / a<k_{z} \leq \pi / a, 0<\varphi \leq 2 \pi$ ). This cylinder is a closed surface in $\boldsymbol{k}$ space (a torus) within which the band structure exhibits a band gap, so that a gap Chern number (sum over all bands below the gap) is well defined. The change in gap Chern number $|\Delta C|$ across an interface equals the number of topologically protected surface states that connect the bulk bands below the gap with those above $[10,11]$. The gap Chern number for the above torus and a hyperconic band at a PWP is given by $|C|=2$, as shown above (note that only the Chern number of the PWP at the gap frequency needs to be considered, as contributions from possible point degeneracies at smaller frequencies cancel). This results in 8 surface bands for the supercell geometry with two $|\Delta C|=4$ interfaces, as reproduced in the inset in Fig. 3(a) (we only show the semicircle with 4 surface bands because of symmetry): each of these bands touches and connects the projected bulk bands above and below the gap and thus is, veritably, protected. The Fermi arclike nature (cf. Ref. [40]) of the topologically protected bands is illustrated in the isofrequency representation in Fig. 4: from a practical perspective, this representation shows the photonic states available to a narrow band emitter at the same frequency. The abundance of surface states in all directions with relatively

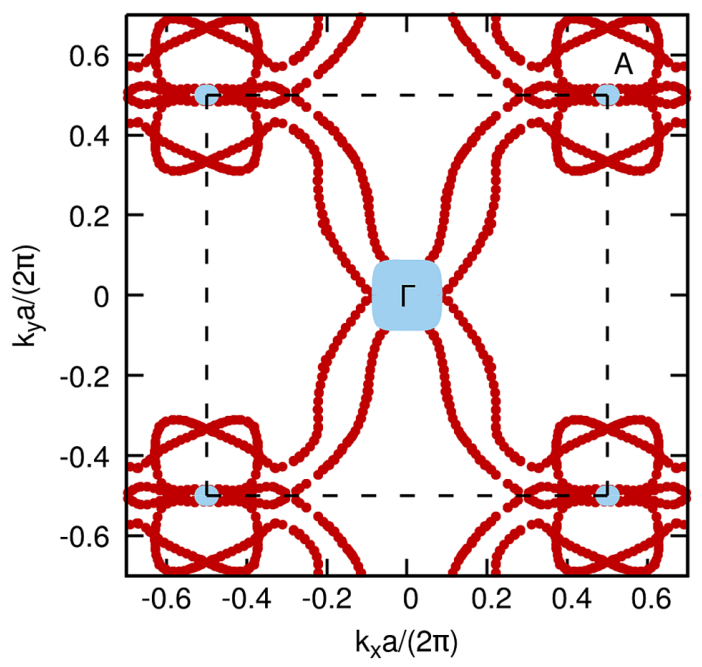

FIG. 4. Isofrequency plot at $k_{0} a /(2 \pi)=0.51$ for the same supercell configuration as in Fig. 3. Bulk modes (blue area) exist only in the direct vicinity of the PWP at $\Gamma$ and the double Weyl cone at $A$. Eight Fermi arclike protected surface bands (red points) emanate from the PWP with topological charge of $C=2$ and connect it with the double Weyl cone that carries the opposite charge $C=-2$. 
small group velocity, whose presence is topologically protected and hence insensitive to fabrication imperfections, suggests that such an emitter will exclusively radiatively decay into surface modes, making PWP systems an ideal starting point to explore topological lasing applications [41-43] in three dimensions.

In this Letter, we have shown that isotropic hyperconic dispersion can be found at the $\Gamma$ point of chiral cubic lattices for a broad class of physical systems. The associated pseudo Weyl points share most features with genuine Weyl points, and have the topological characteristics of a double Weyl point. While the existence of pseudo-Weyl points is deterministic, the magnitude of the slope depends on the particular problem. A desired hypercone can thus be engineered by variation of symmetry preserving system parameters. A natural application exploiting the unique dispersion behavior of these PWPs are zero refractive index materials that have been suggested previously in the context of accidental Dirac points in two-dimensional photonic crystals [19]. We have further demonstrated that the nontrivial topology leads to a number of topologically protected surface states that will be useful in applications such as 3D cavity-free topological lasing.

This work was supported by the EPSRC through program grant EP/L027151/1. We would like to thank Mathew Elman for kindly sharing his illustration of the irreducible Brillouin zone in Fig. 2, and Dr. Paloma Arroyo Huidobro, Gleb Siroki, and Professor Sir John Pendry for helpful discussions.

*m.saba@imperial.ac.uk

†o.hess@imperial.ac.uk

[1] K. v. Klitzing, G. Dorda, and M. Pepper, Phys. Rev. Lett. 45, 494 (1980).

[2] D. J. Thouless, M. Kohmoto, M. P. Nightingale, and M. den Nijs, Phys. Rev. Lett. 49, 405 (1982).

[3] Y. Hatsugai, Phys. Rev. B 48, 11851 (1993).

[4] M. Kohmoto, Ann. Phys. (N.Y.) 160, 343 (1985).

[5] X.-L. Qi and S.-C. Zhang, Rev. Mod. Phys. 83, 1057 (2011).

[6] M. Z. Hasan and C. L. Kane, Rev. Mod. Phys. 82, 3045 (2010).

[7] J. Hofmann and S. Das Sarma, Phys. Rev. B 93, 241402 (2016).

[8] G. Siroki, D. Lee, P. D. Haynes, and V. Giannini, Nat. Commun. 7, 12375 (2016).

[9] M. Onoda, S. Murakami, and N. Nagaosa, Phys. Rev. Lett. 93, 083901 (2004).

[10] S. Raghu and F. D. M. Haldane, Phys. Rev. A 78, 033834 (2008).

[11] L. Lu, J. D. Joannopoulos, and M. Soljačić, Nat. Photonics 8, 821 (2014).

[12] L. Lu, L. Fu, J. D. Joannopoulos, and M. Soljačić, Nat. Photonics 7, 294 (2013).
[13] Q. Lin, M. Xiao, L. Yuan, and S. Fan, Nat. Commun. 7, 13731 (2016)

[14] J. Lu, C. Qiu, S. Xu, Y. Ye, M. Ke, and Z. Liu, Phys. Rev. B 89, 134302 (2014).

[15] Y. D. Chong, X.-G. Wen, and M. Soljačić, Phys. Rev. B 77, 235125 (2008).

[16] B. J. Wieder, Y. Kim, A. M. Rappe, and C. L. Kane, Phys. Rev. Lett. 116, 186402 (2016).

[17] B. Bradlyn, J. Cano, Z. Wang, M. G. Vergniory, C. Felser, R. J. Cava, and B.A. Bernevig, Science 353, aaf5037 (2016).

[18] C. Bradley and A. Cracknell, The Mathematical Theory of Symmetry in Solids: Representation Theory for Point Groups and Space Groups, Oxford Classic Texts in the Physical Sciences (Oxford University Press, New York, 2010).

[19] X. Huang, Y. Lai, Z. H. Hang, H. Zheng, and C. T. Chan, Nat. Mater. 10, 582 (2011).

[20] E. Kane, J. Phys. Chem. Solids 1, 249 (1957).

[21] International Tables for Crystallography, Volume A: Space Group Symmetry, edited by T. Hahn (Springer, New York, 2002), Vol. A.

[22] See Supplemental Material at http://link.aps.org/ supplemental/10.1103/PhysRevLett.119.227401 for a detailed derivation of the pseudo-Weyl Hamiltonian and its Chern characteristics, and for a rigorous theory of the plasmonic sphere packing.

[23] M. Saba, M. D. Turner, K. Mecke, M. Gu, and G. E. Schröder-Turk, Phys. Rev. B 88, 245116 (2013).

[24] M.S. Dresselhaus, Group Theory (Springer, New York, 2013).

[25] C. Herring, Phys. Rev. 52, 361 (1937).

[26] F. G. Frobenius, Gesammelte Abhandlungen I (German Edition) (Springer, New York, 1968).

[27] T. Kato, Perturbation Theory for Linear Operators (Springer-Verlag, Berlin, New York, 1976).

[28] Y. D. Burago and V. A. Zalgaller, Geometric Inequalities (Springer-Verlag, Berlin, New York, 1988).

[29] P. P. Ewald, Ann. Phys. (Berlin) 369, 253 (1921).

[30] J. Hove and J. A. Krumhansl, Phys. Rev. 92, 569 (1953).

[31] M. G. Silveirinha and C. A. Fernandes, IEEE Trans. Antennas Propag. 53, 347 (2005).

[32] G. Weick, C. Woollacott, W. L. Barnes, O. Hess, and E. Mariani, Phys. Rev. Lett. 110, 106801 (2013).

[33] J. Joannopoulos, S. Johnson, J. Winn, and R. Meade, Photonic Crystals: Molding the Flow of Light (Princeton University Press, Princeton, NJ, 2008).

[34] W.-J. Chen, M. Xiao, and C. T. Chan, Nat. Commun. 7, 13038 (2016).

[35] D. Han, Y. Lai, J. Zi, Z.-Q. Zhang, and C. T. Chan, Phys. Rev. Lett. 102, 123904 (2009).

[36] B. T. Draine and P. J. Flatau, J. Opt. Soc. Am. A 11, 1491 (1994).

[37] S. G. Johnson and J. D. Joannopoulos, Opt. Express 8, 173 (2001).

[38] W. Setyawan and S. Curtarolo, Comput. Mater. Sci. 49, 299 (2010).

[39] X. Wan, A. M. Turner, A. Vishwanath, and S. Y. Savrasov, Phys. Rev. B 83, 205101 (2011). 
[40] J. Noh, S. Huang, D. Leykam, Y. D. Chong, K. P. Chen, and M. C. Rechtsman, Nat. Phys. 13, 611 (2017).

[41] G. Harari, M. A. Bandres, Y. Lumer, Y. Plotnik, D. N. Christodoulides, and M. Segev, in Conference on Lasers and Electro-Optics (Optical Society of America, Washington, DC, 2016), p. FM3A.3.
[42] B. Bahari, A. Ndao, F. Vallini, A. El Amili, Y. Fainman, and B. Kanté, Science 358, 636 (2017).

[43] St-Jean, V. Goblot, E. Galopin, A. Lemaître, T. Ozawa, L. Le Gratiet, I. Sagnes, J. Bloch, and A. Amo, Nat. Photonics 11, 651 (2017). 